\title{
Deuteron Flow in Ultrarelativistic Heavy Ion Reactions
}

\author{
R. Mattiello, A. Jahns, H. Sorge, H. Stöcker, and W. Greiner \\ Institut für Theoretische Physik, J. W. Goethe Universität, D-60054 Frankfurtam Main, Germany \\ (Received 13 January 1994; revised manuscript received 22 November 1994)
}

\begin{abstract}
Deuteron momentum distributions are predicted for nucleus-nucleus reactions at beam energies of $(10-15) A \mathrm{GeV}$. The deuteron transverse momentum spectra exhibit a pronounced shoulder-arm shape deviating markedly from thermal distributions due to collective transverse nuclear flow. A clear "bounceoff" event shape is seen for protons and deuterons: The transverse momentum components in the reaction plane are for deuterons up to a factor of 2 larger than for protons. The strength of the collective matter flow is sensitive to the type of baryon potential interaction employed. This allows the study of the transient mean fields at high density in these reactions via the event shape analysis of nucleons and nucleon clusters.
\end{abstract}

PACS numbers: $25.75 .+\mathrm{r}$

The creation and study of strongly interacting matter at high net baryon density has received much attention recently with the opportunity to explore massive reactions at $10 A \mathrm{GeV}$ at the Brookhaven Alternating Gradient Synchrotron (AGS) booster facility. An observable consequence of the formation of dense nuclear matter-far beyond the ground state - is the emergence of collective flow driven by compression-induced pressure [1-4]. For nuclear clusters - as compared to light hadrons - flow can even dominate the momentum spectra [3].

Mean fields [1] may give important contributions to this pressure and could - via the flow effect-be accessible to experimental observation just as in the $1 \mathrm{GeV}$ region [5]. At $\rho_{B}=\rho_{0}$, the nuclear mean field can be decomposed into an attractive scalar field and a repulsive vector potential [6] which is in accord with Dirac phenomenology for optical potential calculations in $p+A$ reactions $[7-10]$. At higher densities the momentum dependence $[7,11]$, the excitation into resonances [12], and the transition to quark matter [13] are expected to play a crucial role. Several new ideas are currently under active investigation: Medium properties of hadrons (e.g., of the $\omega$ meson that is responsible for vector repulsion [14]) or quark and gluon condensates, which break the approximate scale and chiral symmetry of the QCD Lagrangian in the vacuum, could modify the scalar field essentially [13-15].

Beam energies between $10 A$ and $15 A \mathrm{GeV}$, as studied experimentally at the BNL AGS [16-20], seem to be well suited to stop two heavy ingoing nuclei and to create the desired high baryon densities. This has been shown by transport calculations based on hadronic excitations and rescattering like the RQMD approach (strings, resonances) [21,22] or the ARC model (resonances) [23]. The observation of stopping in the AGS experiments has been unclear for quite some time. However, all experimental groups now confirm [16-19] the predicted large baryon stopping in central collisions [21,24].

In this Letter we combine cluster coalescence and the investigation of collective nuclear matter flow. Further- more, final observables - rapidity distributions, $m_{t}$ spectra, and directed flow $p_{x}(y)$ - are compared for two extreme scenarios: one with a Skyrme-type potential between baryons, and the other without (cascade). Here all $\mathrm{Au}(11.6 \mathrm{~A} \mathrm{GeV}) \mathrm{Au}$ results are calculated for central impact parameters $b<3 \mathrm{fm}$.

The relativistic quantum molecular dynamics approach (RQMD 1.07) [21] employed for the calculations presented compares well with experimental single particle and two-body correlation data $[17,19,22]$. It combines the classical propagation of particles with the excitation of hadrons into resonances and strings. Secondaries (emerging from the decaying resonances and strings) undergo subsequent interactions, both with each other and with the ingoing baryons.

RQMD calculations do not contain cluster states (e.g,, deuterons) dynamically. The formation probability of deuterons can be calculated by projecting the generated classical neutron-proton phase-space distribution on the deuteron wave function via the Wigner-function method [25]. This method was applied to bombarding energies around $1 A \mathrm{GeV}$, in combination with the intranuclear cascade model [26] and the QMD [11]. The number of deuterons is given by

$$
d N_{d}=\frac{1}{2} \frac{3}{4}\left\langle\sum_{i_{n}, j_{p}} \rho_{d}^{W}(\Delta \mathbf{x}, \Delta \mathbf{p})\right\rangle d^{3}\left(p_{i_{n}}+p_{j_{p}}\right)
$$

The brackets indicate event averaging. $\rho_{d}^{W}$ is the Wigner density of the Hulthén deuteron wave function. The sum runs for each event over all neutron-proton pairs $\left(i_{n}, j_{p}\right)$ with distance vectors in position $\Delta \mathbf{x}$ and momentum $\Delta \mathbf{p}=\frac{1}{2}\left(\mathbf{p}_{i_{n}}-\mathbf{p}_{j_{p}}\right)$ taken at equal time in the twonucleon rest frame (c.m.s.) immediately after both nucleons have frozen out. The factors $\frac{1}{2}$ and $\frac{3}{4}$ take into account isospin projection and spin averaging. Higher mass fragments are by construction contained in the calculated number of deuterons [2,26]. The number of $A>2$ fragments is small, however, for rapidity values $\mid y-$ $y_{\text {mid }} \mid<1$. 
Figure 1 predicts deuteron rapidity distributions for the reactions $\mathrm{Si}+\mathrm{Si}, \mathrm{Si}+\mathrm{Cu}$, and $\mathrm{Si}+\mathrm{Au}$ at $14.5 A \mathrm{GeV}$ and $\mathrm{Au}+\mathrm{Au}$ at $11.6 A \mathrm{GeV}$. For the $\mathrm{Au}(11.6 A \mathrm{GeV}) \mathrm{Au}$ system $(b<3 \mathrm{fm})$ deuterons are shown together with protons and preliminary data from Ref. [16]. The baryon distributions for $\mathrm{Au}+\mathrm{Au}$ are peaked at midrapidity which proves strong nuclear stopping [24]. The transverse momenta are shown in Fig. 2 for $\mathrm{Au}(11.6 \mathrm{~A} \mathrm{GeV}) \mathrm{Au}$ only. The apparent temperatures in calculations with (without) potentials are extracted from a Boltzmann fit $[d N \propto$ $m_{t}^{2} \exp \left(m_{t} / T\right) d m_{t}$ for $\left.m_{t}-m_{0}>300 \mathrm{MeV}\right]$ and show different values for deuterons $T \simeq 250 \mathrm{MeV}(\simeq 225 \mathrm{MeV})$ and protons $T \simeq 200 \mathrm{MeV}(\simeq 185 \mathrm{MeV})$. The correlation between rapidity and directed transverse momentum $p_{x}(y)$ in Fig. 2(b) is well known as the nuclear bounceoff discovered first at the LBL Bevalac [27]. It reflects collective sideward flow predicted by hydrodynamics and microscopic models [1,24,28].

The RQMD results are not consistent with a thermal fireball or coalescence in momentum space [29-31] which predict similar slope parameters for deuterons and protons [31]. Furthermore, the calculated $d / p$ ratio in calculations with potentials increases in the central

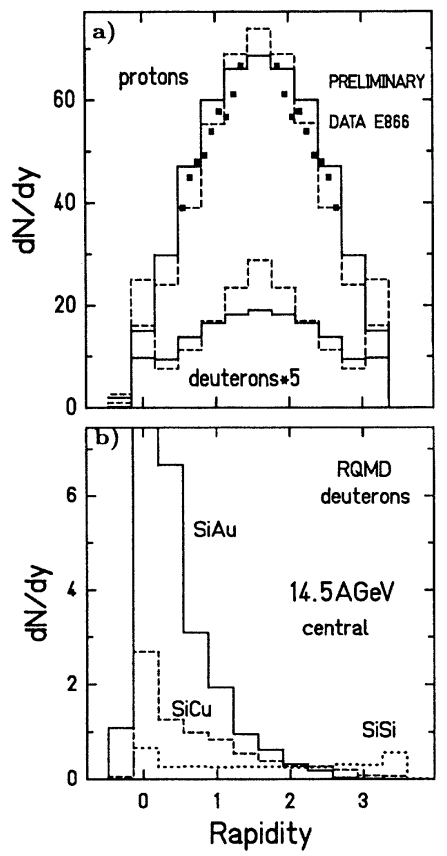

FIG. 1. (a) Prediction of the deuteron and proton rapidity distributions for the reaction $\mathrm{Au}(11.6 \mathrm{~A} \mathrm{GeV}) \mathrm{Au}(b<3 \mathrm{fm})$. Solid histograms denote calculations with potentials, the cascade results are shown by the dashed histograms. The proton spectra are corrected for the coalesced deuterons. The square symbols represent preliminary data for central (4\% ZCAL) $\mathrm{Au}+\mathrm{Au}$ collisions at $11.6 \mathrm{~A} \mathrm{GeV}$ which include approximately $15 \%$ systematic uncertainty [16]. (b) Prediction of the deuteron rapidity distributions in the reactions $\mathrm{Si}+\mathrm{Si}$ (solid), $\mathrm{Si}+\mathrm{Cu}$ (dashed), and $\mathrm{Si}+\mathrm{Au}$ (dotted) at $14.5 \mathrm{~A} \mathrm{GeV}$ incident beam energy calculated with potentials. The impact parameter ranges have been chosen as $b<1 \mathrm{fm}, b<1.5 \mathrm{fm}$, and $b<3 \mathrm{fm}$, respectively.
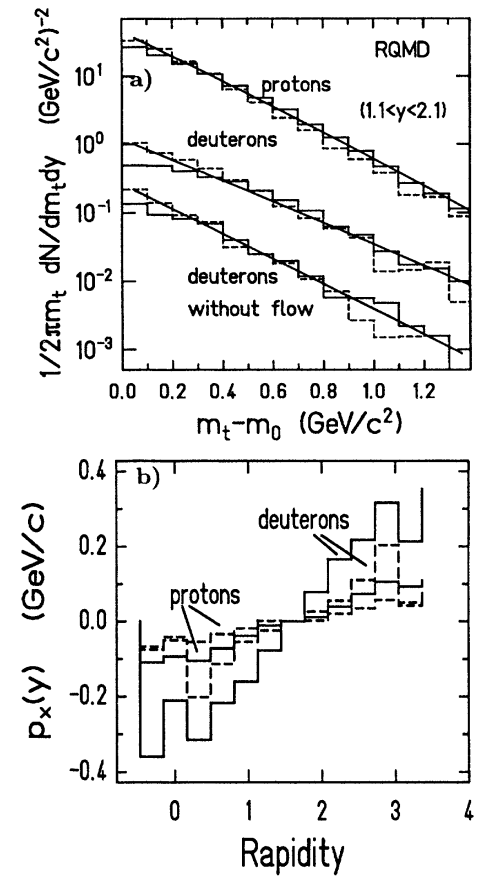

FIG. 2. (a) Prediction of the invariant transverse mass spectra of protons and deuterons at midrapidity $(1.1<y<2.1)$ for the reaction $\mathrm{Au}(11.6 \mathrm{~A} \mathrm{GeV}) \mathrm{Au}, b<3 \mathrm{fm}$ in calculations with (solid histograms) and without (dashed histograms) potentials. In addition, the lower histograms show the deuteron distributions with artificially destroyed momentum-position correlations. (b) The $p_{x}(y)$ correlation for protons and deuterons calculated with (solid histograms) and without (dashed histograms) potentials.

rapidity interval $y_{\text {c.m.s. }} \pm 0.5$ from $\simeq(3.6 \pm 0.3) \%$ for $\mathrm{Si}+\mathrm{Si}$ to $\simeq(5.6 \pm 0.3) \%$ for $\mathrm{Au}+\mathrm{Au}$. In contrast, the scaling with total mass (or volume) in the fireball model (for given freeze-out density, temperature in chemical equilibrium) exhibits equal values [31].

We demonstrate first that collective flow is responsible for the particular momentum spectra of deuterons and protons at midrapidity: The average transverse freezeout velocities of protons [Fig. 3(a)] and the freeze-out density profiles of protons and deuterons [Fig. 3(b)] are calculated as functions of the transverse distance to the beam axis. High baryon densities in the reaction center prevent particles from freeze-out and the induced pressure pushes them outside into the vacuum. This is demonstrated by the difference between the initial (dotted histogram) and final freeze-out positions of protons. The characteristic decrease of the freeze-out profile for $r_{t}<$ $6 \mathrm{fm}$ is more pronounced for deuterons. For protons it becomes stronger with a longitudinal cut in the positions.

The considerable matter flow in Fig. 3 is reflected already in the inclusive spectra: First focus on the low- $m_{t}$ part of the transverse mass spectra in Fig. 2(a). Neglecting statistical fluctuations the transverse momentum of a nucleon is a function of velocity and the freeze-out 


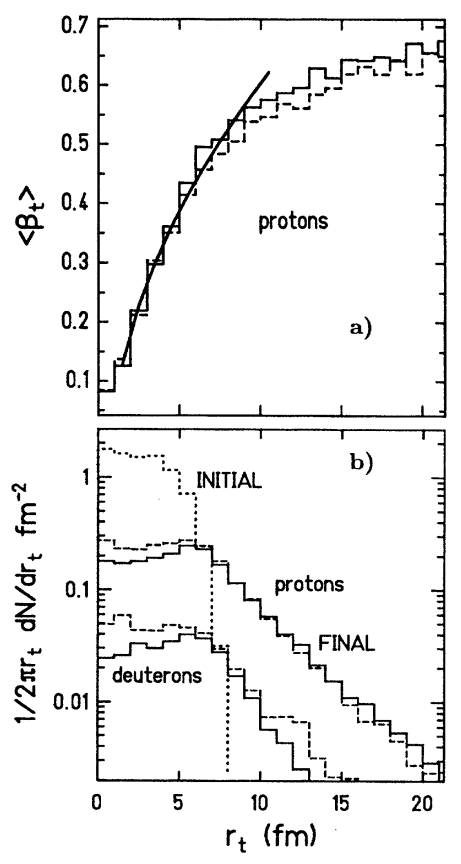

FIG. 3. Freeze-out profiles of protons and deuterons with $1.1<y<2.1$ in the reaction $\mathrm{Au}(11.6 \mathrm{~A} \mathrm{GeV}) \mathrm{Au}$. (a) Transverse velocity profile for protons in calculations with (solid histogram) and without (dashed histogram) potentials. $r_{t}$ is the transverse distance to the beam axis in the c.m.s. frame of the reaction. The solid line shows an analytical approximation for $r_{t}<10 \mathrm{fm}$ of the form $\beta_{t}=A\left(r_{t}-r_{0}\right)^{B}, B=0.55$. (b) Distribution of transverse freeze-out distances for protons and deuterons in calculations with (solid histograms) and without (dashed histograms) potentials. The dotted histogram shows the initial positions of those protons that are contained in the final distributions.

profile only. An effective parametrization $\beta_{t}=$ $A\left(r_{t}-r_{0}\right)^{B}$ for the low- $p_{t}$ part $\left(r_{t}<10 \mathrm{fm}\right)$ of the velocity profile in Fig. 3(a) leads to

$$
\frac{d N}{m_{t} d m_{t}}=\frac{m_{0}^{2}}{B A^{2 / B}} \frac{\left(m_{t}^{2}-m_{0}^{2}\right)^{(1-B) / B}}{\left(m_{t}^{2}\right)^{(1+B) / B}} \rho\left(r_{t}\right),
$$

where $\rho=1 /\left(r_{t}-r_{0}\right) d N / d r_{t}$. With $\rho=$ const the spectrum has a maximum at finite $m_{t}$ only for $(B<1)$ and has a convex shape - consistent with the RQMD results. The $B$ value extracted from the RQMD calculation is about 0.55 . Note that a quadratic dependence $(B=2)$ yields an overall concave spectrum, diverging for $m_{t}-m_{0} \rightarrow 0$. This property has been used to interpret the low- $p_{t}$ pion enhancement in terms of a spherically expanding thermal fireball [32]. A combined analysis of pion and nucleon (cluster) spectra should elucidate the role of collective flow with respect to final transverse momentum spectra.

Second, the large values of $\beta_{t}$ in the region where most of the baryons freeze-out cause a slope splitting between deuterons and protons: The splitting and the strong shoulder-arm curvatures vanish when the flow correlations are destroyed by a random permutation of the final momenta [dashed histogram in Fig. 2(a)]. The absolute yield decreases considerably, i.e., collective flow enhances the probability to find two nucleons in the same phase-space volume. Hence, the predicted increase of the $d / p$ ratio from $\mathrm{Si}+\mathrm{Si}$ to $\mathrm{Au}+\mathrm{Au}$ reflects stronger flow in the heavier system.

We remark that the flow velocities of nucleons and deuterons are similar. Though, the $p_{x}(y)$ correlation in Fig. 2(b) shows roughly a factor 3-4 higher values for deuterons. This effect is caused by the particular interplay of phase-space densities and collective flow velocities which can lead to deuteron momenta larger than the simple scaling with mass number $A$.

Is the collective flow only due to collision induced pressure or does it depend on the transient mean fields at high density? In this Letter we compare two schematic cases: In the first case the potentials are switched off (i.e., the cascade mode is used). The second scenario uses potential type interactions which define effective baryon masses in a medium [21]

$$
p_{i}^{2}-m_{i}^{2}-V_{i}=0
$$

and thus simulate the effect of mean fields. Here

$$
\left(2 m_{N}\right)^{-1} V_{i}=+\frac{1}{2} \sum_{j, j \neq i} \alpha_{i j}\left(\frac{\rho_{i j}}{\rho_{0}}\right)+\frac{\beta}{\gamma+1}\left[\sum_{j, j \neq i}\left(\frac{\rho_{i j}}{\rho_{0}}\right)\right]^{\gamma},
$$

with $\rho_{i j}$ a Gaussian of the c.m.s. distance vector normalized to $1, \rho_{0}$ the ground state matter density, and $\alpha=-0.4356 \mathrm{GeV}, \beta=0.385 \mathrm{GeV}, \gamma=\frac{7}{6}$ parameters which are adjusted to the saturation properties of nuclear matter (binding energy and compressibility). The fit was done by taking the expectation value of the total energy per nucleon for idal gas (plane) wave functions and taking into account antisymmetrization effects. As has been stated in [33], the experimental data for nucleus-nucleus reactions at $(10-15) A \mathrm{GeV}$ seem to indicate more repulsion than that given by a pure density dependence, as in Eq. (3). This additional repulsion is probably caused by the internal excitations of the dense matter produced, i.e., a momentum dependence of nuclear forces. Since the interwining effects of momentum and density dependence are not well understood, we refrain here from assuming a more or less arbitrary parametrization of the momentum dependence. Instead we use the same approach as in [33], i.e., we harden the density dependence of the potentials in order to get agreement with proton singles spectra. [This is achieved by switching off the attractive two-body force in the $\Delta \Delta$ and $N B^{*}$ channel, $\alpha_{\Delta \Delta}=\alpha_{N B^{*}}=0$, thus explaining the index pair $(i j)$ in Eq. (3).] We feel some justification in this approach, because we are mainly interested here in cluster flow.

The results show higher transverse [Fig. 1(a)] and longitudinal [Fig. 2(a)] momenta caused by the additional pressure which is built up by the repulsive mean fields at high baryon density (up to $8 \rho_{0}$ is achieved [24]). Note that the region at most compression $\left(\rho / \rho_{0}>3\right)$ is large $\left(V \simeq\right.$ several hundred $\left.\mathrm{fm}^{3}\right)$ and contains up to $60 \%$ 
baryons in resonance states. The rapidity distribution is flattened [Fig. 1(a)] and the average transverse momentum at midrapidity increases in calculations with potentials [Fig. 2(a)]. With respect to the average momenta the potentials play a minor role (on the level of $10 \%$ ). In contrast, the low- $p_{t}$ part of the $m_{t}$ spectra is changed markedly [Fig. 2(a)] and the flow correlation $p_{x}(y)$ shows roughly a factor $1.5-2$ higher values due to the additional sideward push of the mean fields [Fig. 2(b)].

In conclusion, the microscopic model shows that the strong stopping power recently discovered in nucleus-nucleus collisions at $(10-15) A \mathrm{GeV}$ results in observable collective behavior of the stopped baryon-rich matter. Considerable flow $(\langle\beta\rangle \approx 0.5 c)$ develops due to the internal pressure of the dense matter visible in $p_{x}(y)$ correlations, differences between protons and deuterons in the shoulder-arm shapes of the $m_{t}$ spectra, and $d / p$ ratios which depend on the target-projectile combination. All observables show sensitivities to mean fields in the strongly compressed central region. An even more pronounced sensitivity is expected for larger fragments such as $t$ or He. Regarding the bounceoff effect, the first experimental observation at $10 \mathrm{GeV} /$ nucleon has just been announced [34], as well as azimuthally asymmetric particle correlations in the projectile hemisphere [35]. The very recent experimental discovery of collective flow by the E877 Collaboration is most encouraging: The collective flow as manifested in the spectra of deuterons and heavier clusters can provide a very useful tool for the exploration of in-medium properties of dense and highly excited baryonic matter produced with massive heavy ions at the AGS.

This work is supported by BMFT, GSI, and DFG.

[1] H. Stöcker and W. Greiner, Phys. Rep. 137, 278 (1986); H. Kruse, B. V. Jacak, and H. Stöcker, Phys. Rev. Lett. 54, 289 (1985); J. J. Molitoris, J. B. Hoffer, H. Kruse, and H. Stöcker, Phys. Rev. Lett. 53, 899 (1984); G. Buchwald, G. Graebner, J. Theis, J. Maruhn, and W. Greiner, Phys. Rev. Lett. 52, 1594 (1984).

[2] P. Danielewicz and Q. Pan, Phys. Rev. C 46, 2002 (1992); Q. Pan and P. Danielewicz, Phys. Rev. Lett. 70, 2062 (1993); 70, 3523 (1993).

[3] H. Stöcker, A. Ogloblin, and W. Greiner, Z. Phys. A 303, 259 (1981).

[4] J. J. Molitoris, H. Stöcker, H. A. Gustafsson, J. Cugnon, and D. L'Hote, Phys. Rev. C 33, 867 (1986).

[5] K.-H. Kampert, J. Phys. G 15, 691 (1989); H. H. Gutbrod, K. H. Kampert, B. W. Kolb, A. M. Poskanzer, H. G. Ritter, and H. R. Schmidt, Phys. Lett. B 216, 267 (1989).

[6] B.D. Serot and J.D. Walecka, Adv. Nucl. Phys. 15, 1 (1986); J. Theis et al., Phys. Rev. D 28, 2286 (1983).

[7] E. D. Cooper, B. C. Clark, R. Kozack, S. Shim, S. Hama, J. I. Johansson, H. S. Sherif, R. L. Mercer, and B. D. Serot, Phys. Rev. C 36, 2170 (1987).

[8] M. Jaminon, C. Mahaux, and P. Rochus, Nucl. Phys.
A365, 371 (1981).

[9] B. Ter Haar and R. Malfliet, Phys. Lett. B 172, 10 (1986).

[10] T. L. Ainsworth, E. Baron, G. E. Brown, J. Cooperstein, and M. Prakash, Nucl. Phys. A464, 740 (1987).

[11] J. Aichelin, A. Rosenhauer, G. Peilert, H. Stöcker, and W. Greiner, Phys. Rev. Lett. 58, 1926 (1987); J. Aichelin and E. A. Remler, Phys. Rev. C 35, 1291 (1987).

[12] F. de Jong and R. Malfliet, Phys. Rev. C 46, 2567 (1992).

[13] J. Ellis, J. Kapusta, and K. Olive, Phys. Lett. B 273, 122 (1991).

[14] U. Vogl and W. Weise, Prog. Part. Nucl. Phys. 27, 195 (1991); W. Weise, Nucl. Phys. A553, 59 (1993).

[15] X. Jin, T.D. Cohen, R. J. Furnstahl, and D. K. Kriegel, Phys. Rev. C 47, 2882 (1993), and references therein.

[16] E802/E866 Collaboration, M. Gonin, Nucl. Phys. A566, $601 \mathrm{c}(1993)$.

[17] E-802/866 Collaboration, H. Hamagaki, Nucl. Phys. A566, 27c (1993).

[18] E810 Collaboration, K. J. Foley, Nucl. Phys. A554, 335c (1992).

[19] E-814/877 Collaboration, J. Stachel, Nucl. Phys. A566, 183c (1993).

[20] E-814/877 Collaboration, J. Barette, Nucl. Phys. A566, $411 \mathrm{c}(1993)$.

[21] H. Sorge, H. Stöcker, and W. Greiner, Ann. Phys. (N.Y.) 192, 266 (1989); Nucl. Phys. A498, 567c (1989); H. Sorge, A.v. Keitz, R. Mattiello, H. Stöcker, and W. Greiner, Z. Phys. C 47, 629 (1990).

[22] H. Sorge, R. Mattiello, H. Stöcker, and W. Greiner, Phys. Lett. B271, 37 (1991).

[23] S. H. Kahana, Y. Pang, and T.J. Schlagel, Nucl. Phys. A566, 465c (1993), and references therein.

[24] H. Sorge, A. v. Keitz, R. Mattiello, H. Stöcker, and W. Greiner, Phys. Lett. B 243, 7 (1990).

[25] E. A. Remler, Ann. Phys. (N.Y.) 136, 293 (1981), and references therein.

[26] M. Gyulassy, K. Frankel, and E. A. Remler, Nucl. Phys. A402, 596 (1983).

[27] H. A. Gustafsson, H. H. Gutbrod, J. Harris, B. V. Jacak, K. H. Kampert, B. Kolb, A. M. Poskanzer, H. G. Ritter, and H. R. Schmidt, Mod. Phys. Lett. A3, 1323 (1988).

[28] N. S. Amelin, E. F. Staubo, L. P. Csernai, V. D. Toneev, K. K. Gudima, and D. Strottman, Phys. Rev. Lett. 67, 1523 (1991).

[29] H. Gutbrodt, A. Sandoval, P. Johanssen, A. Poskanzer, O. Gosset, W. Meyer, G. Westfall, and R. Stock, Phys. Rev. Lett. 37, 667 (1976).

[30] G. D. Westfall, J. Gosset, P. J. Johansen, A. M. Poskanzer, and W. G. Meyer, Phys. Rev. Lett. 37, 1202 (1976).

[31] A. Mekijan, Phys. Rev. Lett. 38, 640 (1977); Phys. Rev. C 17, 1051 (1978).

[32] K. S. Lee and U. Heinz, Z. Phys. C 48, 525 (1990).

[33] H. Sorge, R. Mattiello, H. Stöcker, and W. Greiner, Phys. Rev. Lett. 68, 286 (1992).

[34] E877 Collaboration, P. Braun-Munzinger, NATO Advanced Studies on Nuclear Physics Summer School, Bodrum, Turkey, 1993.

[35] E-802 Collaboration, T. Abbott et al., Phys. Rev. Lett. 70, 1393 (1993) 